\title{
The Design of an Dispersion-compensating Photonic Crystal Fiber for Optical Transport Network
}

\author{
LEI Hu ${ }^{1, a}$, YIN Shuhua* ${ }^{2, b}$ \\ ${ }^{1}$ Xi'an fanyi university, Xi'an, China \\ Shuhua_y@126.com
}

\begin{abstract}
Keywords: Dispersion Compensation Fiber Photonic Crystal Fiber Dispersion Compensation Error Code Rate DWDM
\end{abstract}

\begin{abstract}
Due to the dispersion compensation characteristic exhibited by the Photonic Crystal Fiber (PCF), the dispersion compensation based PCF for compensating the G.652 fiber was designed in this research so as to meet the business demands of the fiber communication system. The dispersion compensation characteristic of the PCF was analyzed using a Multi-Pole Method (MPM) to obtain change law of the dispersion with the change of structure parameters. Based on the change law, dispersion compensation based W type PCF which includes was designed. The fiber cladding included nine-layer vents with the dispersion value of $-402.7 \mathrm{ps} \cdot \mathrm{km}^{-1} \cdot \mathrm{nm}^{-1}$ and its dispersion slope of $-1.427 \mathrm{ps} \cdot \mathrm{km}^{-1} \cdot \mathrm{nm}^{-2}$ at $1550 \mathrm{~nm}$. Using Optisim platform, a dispersion compensation based DWDM system was simulated. Results demonstrated that bit error rates of the system were all less than $10^{-12}$, which satisfies requirements of the bandwidth communication.
\end{abstract}

\section{Introduction}

The dispersion compensation fiber (DCF) is a commonly used dispersion compensation method. Because the dispersion slope of existing DCF fails to match the traditional fiber completely, dispersion effect cannot be compensated exactly and simultaneously on multiple wavelengths and thus leads to residual dispersion. Therefore, to meet the demands of current multi-channel fiber-optic communication systems, it is press to design the dispersion compensation based PCF, which can compensate the bandwidth limitation of traditional fibers[1].

As Birks et al. from Bath University, UK, put forward the idea of conducting the dispersion compensation on PCF in 1998, the dispersion compensation characteristic of PCF has been a hot research issue.

PCF [2] is a kind of new fiber, whose cladding containing many periodically arranged vents. In recent years, researches have been carried out mostly on changing structure of PCF, and dispersion characteristics of the material: for example, for changing the shape of fiber core for PCF, PCFs with near- rectangle core and snowflake crystal core have been reported respectively [3, 4]; as for the studies into change of vent size of PCF have been conducted, such as, the research of PCF with gradient structure. Among them, the method which is most suitable for the dispersion compensation is to change vent size of the PCF[5, 6]. A. Huttunen et al. designed a dual core and negative DCF by changing the vent on the third layer in 2005. T. Matsui et al. designed a duel claddings and large negative DCF by changing the vents on the first and second layer simultaneously in 2007[7].

Based on the MPF[8] and change law of the dispersion compensation characteristic of PCF, dispersion compensation based PCF was put forward in the paper. Afterwards, an Optisim software was used to simulate the compensation effect of the dispersion compensation based W type PCF in $16 \times 10 \mathrm{Gbit} / \mathrm{s}$ fiber-optic communication systems. The simulating results showed that the dispersion compensation based W type PCF is able to well compensate dispersion of ordinary fibers. 


\section{Design of the dispersion compensation based PCF}

It is known that DCF has to meet its own standard: the large negative dispersion value and big negative dispersion slope. It is considered to be more favorable if it can compensate both dispersion and dispersion slope simultaneously. Therefore, the dispersion compensation based PCF needs to be designed by considering the dispersion value and dispersion slope so as to choose the Dispersioncompensating Photonic Crystal fiber (DCPCE) .

MPM is usually employed for providing theoretical simulation in the design of PCF. MPM is a precise simulating method aiming at the circular structure design of cladding vent, and also an extension method of studying conventional fiber methods.

Within the range of a specific wavelength, the analysis on the dispersion compensation characteristic of the PCF was conducted using MPM by changing the diameter $\mathrm{d}$, and space distance $\Lambda$, of the vent respectively.

Case 1: supposed $\mathrm{d}=0.6 \mathrm{um}$ is constant, and $\Lambda$ is $0.7 \mathrm{um}, 0.9 \mathrm{um}, 1.1 \mathrm{um}$ and $1.3 \mathrm{um}$ respectively, the variations of the dispersion value and slope with the change of the wavelength in the range of 1.4 um $\sim 1.6$ um are shown in Figures 1 and 2 respectively.

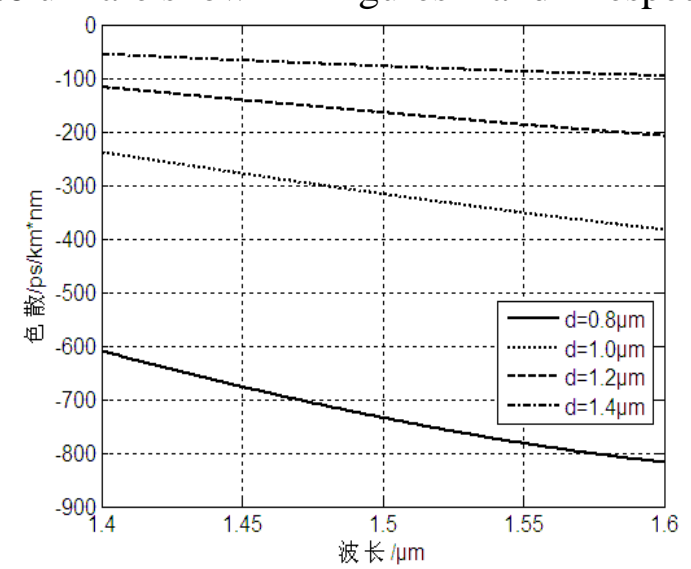

Figure1 The relationship between the wavelength and the dispersion which varies with the change of space distance

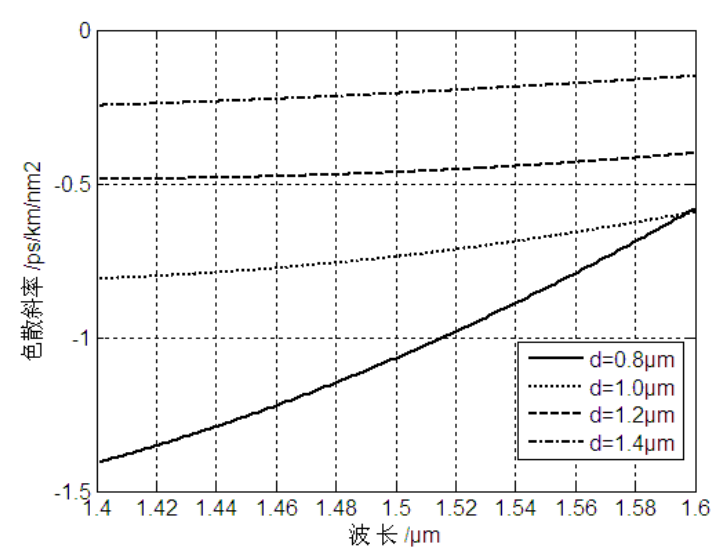

Figure2 The relationship between the wavelength and the dispersion slope with the change of space distance

Case 2; in case $d$ is $0.45 \mathrm{um}, 0.5 \mathrm{um}, 0.6 \mathrm{um}$ and $0.7 \mathrm{um}$ respectively, the variations of the dispersion value, and the slope, with the wavelength in the range of $1.4 \mathrm{um} \sim 1.6 \mathrm{um}$ are indicated in Figures 3 and 4 respectively.

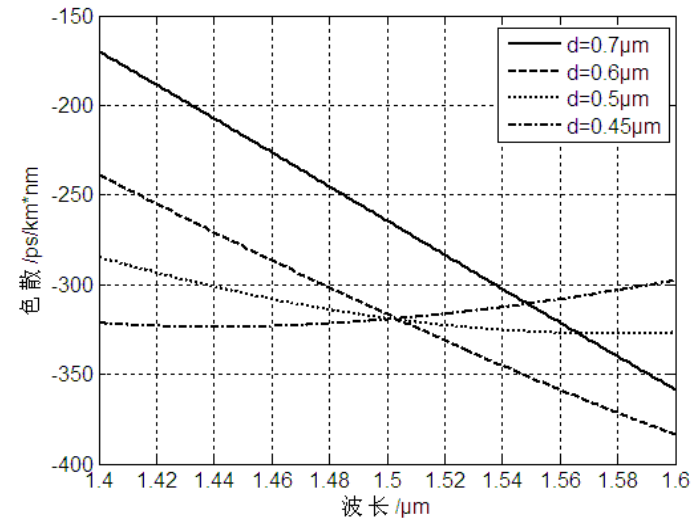

Figure3 The relationship between the wavelength and the dispersion with variation of diameter of the vent

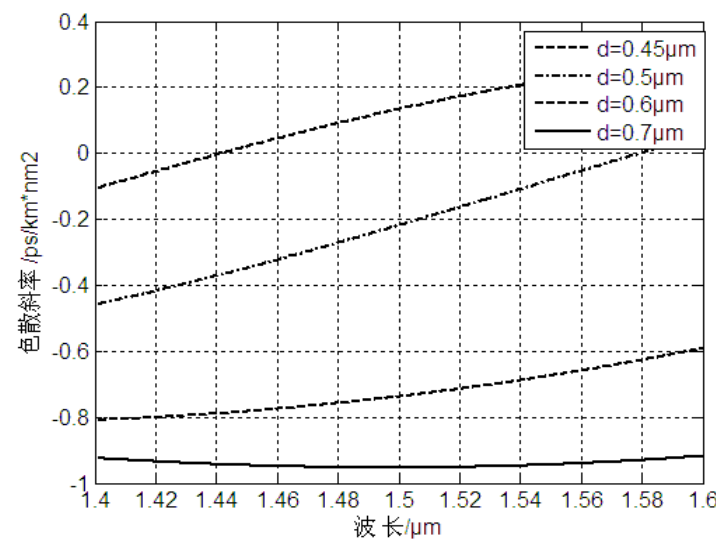

Figure4 The relationship between the wavelength and dispersion slope with the change of vent diameter

As shown in Figures 1and 2, when $d=0.60 \mu \mathrm{m}, \Lambda$ decreases; the dispersion value and the dispersion slope of the PCF at the point $1550 \mathrm{~nm}$ decrease simultaneously. This variation trend is beneficial for 
simultaneously compensating the dispersion value and dispersion slope, which is of great significance for realizing the bandwidth compensation.

As illustrated in Figures 3 and 4, in the case of $\Lambda=1.0 \mu \mathrm{m}$ and decreasing $d$ the dispersion value at $1550 \mathrm{~nm}$ decreases firstly and then increases, while the dispersion slope gradually increases; when $\mathrm{d}=0.45 \mathrm{um}$, the value of the dispersion slope is positive, which is not conducive for compensation on the common fiber. However, according to the variation law of the dispersion of the PCF with the change of its structure, dispersion can be realized by reasonably regulating structural parameter.

According to the analysis aforementioned, the cladding of PCF which is designed for dispersion compensation has nine layer vents. Among which, the sizes of the vents on first, second and third layers are different from that on other layers. The space distance of vent $\Lambda$ in this fiber is $1.0 \mathrm{um}$, the diameters of the vents on the first, second and third layers are $0.76 \mathrm{um} 0.22 \mathrm{um}$ and $0.58 \mathrm{um}$ and the section is shown in Figure 5.

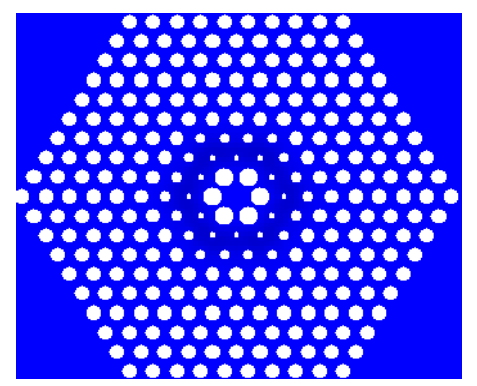

Figure5 The section of the dispersion compensation based PCF

Effective refractive index of the fiber cladding can be calculated directly by using the software Cudos in the following operations. Then, the dispersion and dispersion slope simulated using Matlab are shown in Figure 6.
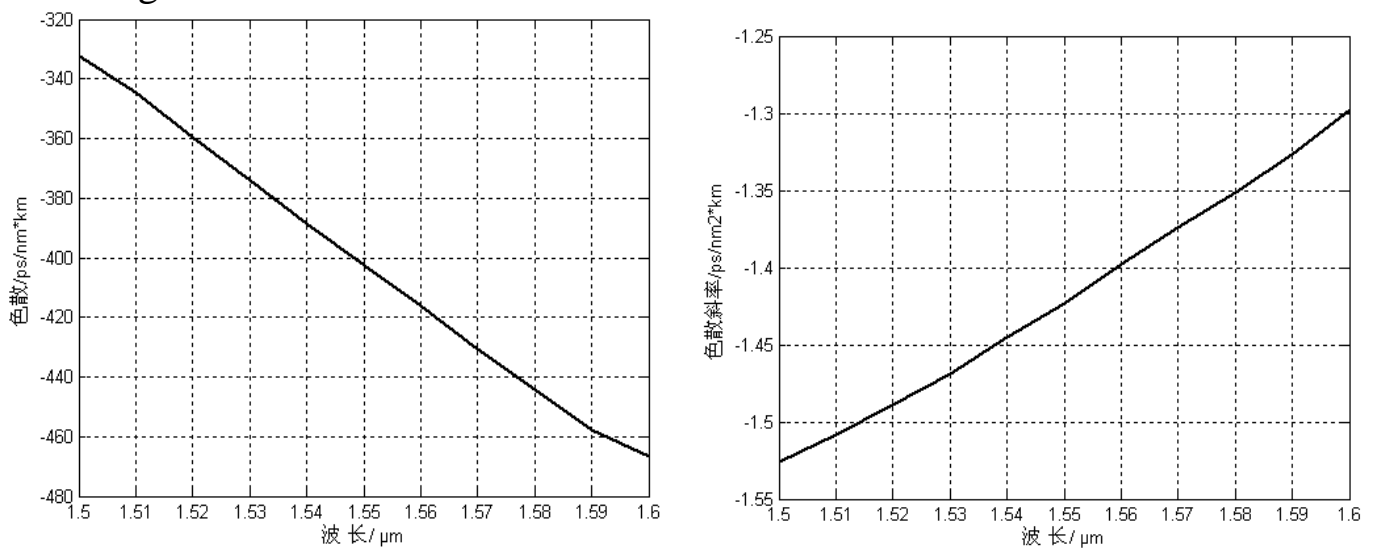

Figure6 Curve relation between wavelength, and dispersion and dispersion slope.

As illustrated in Figure 6, the dispersion value of the fiber and dispersion slope at $1550 \mathrm{~nm}$ are $-402.7 \mathrm{ps} \cdot \mathrm{km}^{-1} \cdot \mathrm{nm}^{-1}$ and $-1.427 \mathrm{ps} \cdot \mathrm{km}^{-1} \cdot \mathrm{nm}^{-2}$ respectively; while Kappa value and the relative dispersion slope are $282.2 \mathrm{~nm}$ and $0.00354 \mathrm{~nm}^{-1}$ respectively. According to the principle of dispersion compensation, this fiber can well compensate the fiber G.652 whose dispersion value and dispersion slope are $16.78 \mathrm{ps} \cdot \mathrm{km}^{-1} \cdot \mathrm{nm}^{-1}$ and $0.0595 \mathrm{ps} \cdot \mathrm{km}^{-1} \cdot \mathrm{nm}^{-2}$ respectively. Meanwhile, the fiber is capable of compensating dispersion value and dispersion slope which are 24 times greater than that of the fiber G.652, which is far higher than the capacity of the common dispersion fiber.

\section{The $16 \times 10 \mathrm{Gbit} / \mathrm{s}$ communication system based on the dispersion compensation based $\mathrm{W}$ type PCF}

Based on the analysis on the dispersion of $\mathrm{W}$ type PCF, the $\mathrm{W}$ type fiber not only meets the demands of the DCF, but also compensate dispersion value and dispersion slope of the fiber G.652 simultaneously. Besides, its compensation efficiency was higher than that of the common DCF. Therefore, a simulation platform was established by using the simulation software OptSim from RSOFT Co, Ltd. 
The system in the simulation platform mainly consists of optical transmitter, wavelength division multiplexer, optical repeater, optical fiber link module and optical receiver. The optical transmitter includes 16 TX1 modules, which is composed of data source, continuous laser and external modulator. The code pattern of the TX1 is NRZ, and transmission rate of each optical beam is Gbit/s. Meanwhile, distance between two intervals in this communication system is set to $0.8 \mathrm{~nm}$ and the wavelength ranges from 1548.51 to $1560.61 \mathrm{~nm}$. The optical signals are firstly coupled in the power amplifier (PA) by a combiner, and after being amplified, they are transmitted into the optical fiber including six segments in total. The lengths of transmission fiber and DCF in each segment are same, which are $4 \mathrm{~km}$ and $96 \mathrm{~km}$ respectively. Besides, the hybrid compensation is adopted as the compensation mode. PA, line amplifier (LP) and pre-amplifier (BA) are adopted in the optical fiber link with the noise amplification coefficient of $4.5 \mathrm{~dB}$. In the receiver, the signals are separated by using optical demultiplexer respectively, and then transmitted to the spectrum analyzer after being processed using filter, so as to observe the bit error rate in each channel. Six communication channels are selected to analyze the system.

Table 1. Bit error rate value of the six communication channels after being compensated in the WDM system

\begin{tabular}{|c|c|c|c|}
\hline wavelength $(\mathrm{nm})$ & 1548.51 & 1550.12 & 1552.52 \\
\hline Error code rate & $1.23618 \times 10^{-16}$ & $3.44612 \times 10^{-15}$ & $3.89224 \times 10^{-14}$ \\
\hline wavelength $(\mathrm{nm})$ & 1555.75 & 1558.17 & 1560.61 \\
\hline Error code rate & $1.49702 \times 10^{-13}$ & $1.35707 \times 10^{-13}$ & $2.57559 \times 10^{-15}$ \\
\hline
\end{tabular}

The simulation results are shown in Table1.Table 1 indicates that this dispersion compensation based PCF realizes the compensation of six channels. In addition, bit error rates are all less than $10^{-12}$, which showing that no signal was distorted. As a result, the expected compensation effect was realized. Application of the PCF does not only save length of DCF, but also fulfill the transmission with very long distance and high capacity. Therefore, it is of great practical and theoretical significance for the future development of the communication system.

\section{Conclusions}

Based on a theoretical analysis on the dispersion compensation characteristic of PCF, a PCF which fits for compensating the optical fiber G.652 was designed. The research simulated the16×10 Gbit/s fiber-optic communication systems of compensating PCF by using the software Optisim. Results demonstrated that the dispersion compensation based W type PCF can be applied in 16×10 Gbit/s communication system which takes G.652 as transmission fiber. Moreover, its compensation efficiency is higher than that of the common DCF. With the continuous improvement of PCF preparation technology, cost and loss decreases. This PCF is expected to play an important role in fiber-optic communication systems in the future.

\section{References}

[1] Birks, D. Mogilevtsev, J.C. Knight, et al. Dispersion Compensation Using Single -Material Fibers[J], Optical Technology .Letters,1999,11(6):674-676.

[2] A. Bjarklev, J. Broeng, A.S. Bjarklev. Photonic Crystal Fibres[J]. Boston:Kluwer Academic Publishers,2003,16(3):103-161.

[3] LiWen Wang, ShuQin Lou, WeiGuo Chen, WenLiang Lu and Xin Wang. Design and Fabrication of Novel Broadband Photonic Crystal Fibers[J].Chinse Journal of Lasers, 2012, 39 : 605008-605012. 
[4] GuoShu qin,LIU Yin ping, ZHU Guang xin,et al. Photonic Crystal Fiber with Snowflake Shape Crystal Core[J]. Acta Photonica Sinica,2007,7(2):1207 1209.

[5] Liwen Wang.Design and Fabrication of Novel Broadband Photonic Crystal Fibers, edited by Beijing Jiaotong University,Beijing,China(2012).

[6] A. Huttunen, P. Torma. Optimization of dual- core and microstructure fiber geometries for dispersion compensationand large mode area [J]. Optics. Express, 2005, 13(2):627 635.

[7] T. Matsui, K. Nakajima, I. Sankawa,et al. Dispersion Compensation Over All the Telecommunication Bands With Double-Cladding Photonic-Crystal Fiber [J]. IEEE Journal Of Lightwave Technology ,2007, 25(3):757 761.

[8] T. White, R. McPhedran, L. Botten, et al. Calculations of Air-Guided Modes in Photonic Crystal Fibers Using the Multipole Method[J]. Optics Express, 2001,9(13):721-732. 